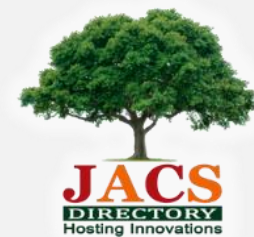

Journal of Nanoscience and Technology

\title{
Biosynthesis and Characterization of Iron Oxide Nanoparticles via Syzygium aromaticum Extract and Determination of Its Cytotoxicity against Human Breast Cancer Cell Lines
}

\author{
T. Thenmozhi1, Nadella Rasajna2 ${ }^{2}$ Megala Rajesh³, N. John Sushma1,* \\ ${ }^{1}$ Department of Biotechnology, Sri Padmavati Mahila Visvavidyalayam, Tirupati - 517 502, Andhra Pradesh, India. \\ ${ }^{2}$ Department of Biological Sciences, IIIT-Srikakulam, Etcherla - 532 410, Andhra Pradesh, India. \\ ${ }^{3}$ Department of Physics, Sri Venkateswara University, Tirupati - 517 502, Andhra Pradesh, India.
}

\section{ART ICLE DETAILS}

Article history:

Received 15 October 2018

Accepted 27 November 2018

Available online 05 January 2019

\section{Keywords:}

Iron Oxide Nanoparticles

Syzygium aromaticum

FT-Raman

Cytotoxicity

\begin{abstract}
A B S T R A C T
Magnetic iron oxide nanoparticles exhibit many interesting properties due to their surface chemistry and they can be exploited in biomedical and technological applications. The iron oxide nanoparticles (FeO NPs) using ferric chloride, ferrous chloride, PVP (Poly Vinyl Pyrolidone) and Syzygium aromaticum extract were successfully synthesized by wet-chemical co-precipitation method. The PVP coated FeO NPs and S. aromaticum loaded to PVP coated FeO NPs were systematically characterized by using expository tools. UV-Visible spectroscopy showed the maximum absorbance at $480 \mathrm{~nm}$ and $554 \mathrm{~nm}$ due to the excitation of surface plasmon vibrations in the FeO NPs formation. Fourier transform infrared (FT-IR) spectrum showed the presence of functional groups of in the form of various band positions and this study confirmed that phenols and primary amines were majorly present. DLS studies revealed narrow size of particles i.e., $399.8 \mathrm{~nm}$ and $190.1 \mathrm{~nm}$. Particle size distribution was showed at $256 \mathrm{~nm}$ and $153 \mathrm{~nm}$ for these samples and zeta potential values were observed at -23.7 and $-18.8 \mathrm{mV}$ with greater stability. The X-ray diffraction (XRD) analysis is in good agreement with a high degree of crystallographic nature of particles and the average particle size was found to be $23 \mathrm{~nm}$ and $29 \mathrm{~nm}$ using Scherrer equation. FT-Raman spectrum showed three main characteristic peaks. The scope of this study was extended by examining the cytotoxicity of PVP coated FeO NPs and S. aromaticum loaded PVP coated FeO NPs on human breast (MCF-7) cancer cell lines. The results suggest that the $S$. aromaticum loaded FeO NPs acts as a potential anti-cancer agent and are a promising research field for lots of biomedical applications particularly for breast cancer studies.
\end{abstract}

\section{Introduction}

Breast carcinoma is the most common prevalent malignancy in women [1]. It is one of the primary causes of morbidity and mortality for women worldwide [2, 3]. In India, breast cancer is the most frequently diagnosed fatal cancers $(21 \%)$. Hence, in order to improve the efficiency of breast cancer therapy and to minimize its harmful impact on healthy organs and tissues, new treatment regimens are urgently needed. A variety of approaches are being practiced for the treatment of cancer each of which has some significant limitations and side effects [4]. The modern treatment for cancers include surgical removal, chemotherapy, radiation and hormone therapy. Conventional chemotherapy fails to target the cancerous cells particularly without interacting with the normal body cells. Thus they cause serious effects resulting in organ damage. In environmental subsistence, there is a need to thrive the environmental friendly strategies to avoid the toxic chemicals in the synthesis protocols in order to circumvent adverse effects in medical applications.

In the ad hoc century, research on nanotechnology advanced much importance in the fields of biology, medicine, physics, chemistry and electronics. It has been the area of interest over the last decade for flourishing precise drug delivery systems as it affords numerous benefits to overwhelm the limitations of conventional formulations [5]. Various researches are being carried out in order to discover more accurate nanotechnology based cancer treatment to minimize the side effects of the conventional ones by using nanoparticles [6]. Noble metal nanoparticles have received unprecedented attention because of their potential efficacy in various fields such as material sciences, pharmaceutical sciences, and biomedical applications $[7,8]$. Iron oxides have mesmerized a plenitude recognition among specialists because of their multifunctional properties such as small size, high magnetism and low toxicity [9] microwave absorption properties, [10] targeted drug delivery in clinical trials [11] and as substrates in cancer treatment methods [12]. The antitumor mechanism could work through the generation of reactive oxygen species or apoptosis and necrosis [13]. And the addition of PVP is widely used in nanoparticle synthesis in which PVP act as stabilizer and is utmost recurrent biologically good-natured polymer due to its diverse biological applications and used as defensive coating for drug delivery. PVP is a bulky, non-toxic [14] non-ionic [15] polymer with $\mathrm{C}=\mathrm{O}, \mathrm{C}-\mathrm{N}$ and $\mathrm{CH}_{2}$ functional groups [16]. The PVP molecule holds a strongly hydrophilic component (the pyrrolidone moiety) and a substantial hydrophobic group (the alkyl group). Ruiz-Baltazar [17] described that a change in the amount of PVP during chemical reduction synthesis of iron oxide nanoparticles can constraint the oxidation of the final nanoparticles as well as change the final morphology. Also, the PVP addition regulates crystal growth, resulting in smaller crystalline sizes, together with limiting the oxidation of the iron oxide nanoparticles.

When coming to the biology nanoparticles are synthesized with the help of medicinal plants as reducing agents and is a captivating research area by synthesizing different types of nanoparticles like iron, copper, gold, silver copper, and zinc [18]. There is a growing demand to flourish ecofriendly methods, which are free from toxic substances, for the synthesis of nanoparticles [19]. The herbal extracts have got many scientific attentions because of its utility in diverse health-beneficial effects such as antidiabetic, anticancer, and antioxidant activities [20]. The natural products procure from the medicinal plants bestow an excellent source of cancer medication. The use of natural products in cancer therapy provide many advantages because in addition to killing the cancer cells, some natural agents have been constitute to render protection to the normal cells [21]. Application of plants and plant products in the treatment of cancer seems to be inevitable, manifest the basis for modern medical science and sustain a great source for new drugs [22]. Syzygium aromaticum (S. aromaticum) (synonym: Eugenia cariophylata) often 
known as clove belongs to family Myrtaceae, native to Indonesia, is a median size tree. The clove is recognized to be beneficial in ethnic systems of medicine. The principal bioactive compound of clove is eugenol, and found in concentrations ranging from 9381.70 to $14650.00 \mathrm{mg}$ per $100 \mathrm{~g}$ of fresh plant material [23]. The phenolic acids and gallic acid are the compounds endow in superior concentration. Flavonoids such as kaempferol, quercetin and its glycosilated groups are also launch in clove. Recently, many reports established the antibacterial, antifungal, antiviral and anticarcinogenic properties of this plant. To the best of our knowledge, no one has been delineate the synthesis of syzygium aromaticum extract loaded to PVP coated iron oxide nanoparticles at room temperature and ascertained their characterization. Hence the present study was designed to evaluate synthesis, characterization and cytotoxic effect of Syzyzium aromaticum loaded PVP coated iron oxide nanoparticles with their unique properties to ameliorate the treatment options available for breast cancer.

\section{Experimental Methods}

\subsection{Chemicals}

Ferrous chloride, Ferric chloride, Sodium hydroxide, Poly Vinyl Pyrrolidine (PVP) were obtained from Himedia. Fluconazole, streptomycin and doxorubicin were obtained from Thermo Fisher Scientifics, India. Breast cancer cell lines (MCF-7) were purchased from NCCS, Pune, India. Delbeccos Modified Eagle's Medium (DMEM) and MTT reagents were purchased from Himedia.

\subsection{Collection of S. aromaticum Flower Buds Material}

Syzygium aromaticum flower buds (Dry clove) were purchased from local grocery store Tirupati, Chittoor District, Andhra Pradesh, India and are dried for 15 to 20 days under shade to vaporize residual moisture and finally ground with blender for further use.

\subsection{Preparation of Cloves Extraction}

The cloves powder was subjected to soxhlet extraction $\left(70 \pm 10^{\circ} \mathrm{C}\right)$ with methanol as a solvent in 1: $4(\mathrm{w} / \mathrm{v})$ ratio for $18 \mathrm{hrs}$. The extract was concentrated using a rotary vacuum evaporator $\left(40{ }^{\circ} \mathrm{C}\right.$; pressure $70 \pm 5$ psi); thick, brown mass obtained was kept in vacuum desiccator for complete drying. The dried extract was stored in an airtight container and kept in the refrigerator $\left(8 \pm 2^{\circ} \mathrm{C}\right)$, and the same was used for in vitro studies.

\subsection{Synthesis of Iron Oxide Nanoparticles}

$8 \mathrm{~mL}$ of $1 \mathrm{M}$ ferric chloride and $2 \mathrm{~mL}$ of $2 \mathrm{M}$ ferrous chloride were mixed and stirred well. While stirring $\mathrm{NaOH}$ solution was added drop by drop until the solution turns brown. After preparing iron oxide nanoparticles, 5 g of PVP was added and sonicated for 30 minutes. Then, the miscellany was heated for 30 minutes at $600{ }^{\circ} \mathrm{C}$.

\subsection{Loading of Drug (Syzygium aromaticum Extract) into PVP-Coated Iron} Oxide Nanoparticles

The drug loading was carried out by dispersing $5 \mathrm{mg}$ of PVP-coated iron oxide nanoparticles in $5 \mathrm{~mL}$ of Syzygium aromaticum extract solution. The mixture of PVP-coated iron oxide nanoparticles with herbal drug can be shaken in a rotary shaker $(200 \mathrm{rpm})$ at $37^{\circ} \mathrm{C}$ for $24 \mathrm{hrs}$. The herbal drug loading can be determined as the difference between the initial drug concentration and the drug concentration in the supernatant. Then these magnetic nanoparticles with drug are magnetically separated and dried.

UV Abs. drug - UV Abs. of the supernatant

Loading efficiency $(\%)=$ x100

UV Abs. of drug

\subsection{Characterization}

\subsubsection{UV-Visible Spectroscopy}

The UV/Vis spectra of surface plasmon resonance of iron oxide nanoparticles were analyzed using Hitachi - 2900 spectrophotometer (Model no. 2J1-0004). The range of measurement is taken from 200 to 800 $\mathrm{nm}$. Blanks were prepared with distilled and deionized (DI) water using a Milli-Q water purification system (Millipore Corp.).

\subsubsection{Fourier Transform Infra-Red (FTIR) Spectroscopy}

The FTIR measurements were performed on a Bruker Alpha-T-109974, SN-200220. FTIR spectrometer (Bruker, Karlsruhe, Germany) equipped with a mid-infrared source (SiC) and a thermal detector, such as deuterium https://doi.org/10.30799/jnst.178.19050103 oxide, L-alanine doped triglycine sulfate premium performance pyroelectric detector. FTIR Spectra were measured in between 500 to $3500 \mathrm{~cm}^{-1}$ region by mixing the samples with $\mathrm{KBr}$ powder. FTIR spectroscopy measurements were perpetrate to recognize the chemical functional group in the sample.

\subsubsection{Particle Size Analysis and Zeta Potential}

The mean particle size and zeta potential of PVP coated iron oxide nanoparticles and drug-loaded PVP-coated iron oxide nanoparticles were estimated using a particle size analyzer (Horiba Scientific Nanopartica, SZ100Z) to know the average size and stability of particles. Particle size analyzer is used to characterize the size distribution of particles in a given sample.

\subsubsection{Dynamic Light Scattering (DLS)}

The mean hydrodynamic particle size distribution and diameter was obtained by DLS. DLS measurements were performed using a zeta sizer (VER.7.11. Serial No: MAL1062727, Malvern Instruments UK). The samples were measured at $25^{\circ} \mathrm{C}$. The results were conferred at intensity and number-based distributions.

\subsubsection{XRD Analysis}

The PVP coated iron oxide nanoparticles and $S$. aromaticum loaded to PVP coated iron oxide nanoparticles were investigated using X-ray diffractometer (XRD-6000, SHIMADZU, Japan), it was used to analyze crystalline nature and calculate the average size of particles.

\subsubsection{FT-Raman Studies}

Raman spectroscopy is widely used to analyze the structural and chemical properties of the synthesized iron oxide nanoparticles and also used to measure the vibration modes of the molecules. The synthesized nanoparticles were recorded with degree of graphitization by Raman spectrophotometer using $16+\mathrm{Mw}(\mathrm{srl}=10)$ laser power.

\subsubsection{Cytotoxicity Assay}

Cytotoxicity assay measures the killing capacity of test compound against a target cell. One of the common methods to determine cytotoxicity is by MTT (3-[4,5-dimethylthiazol-2-y l]-2,5 diphenyltetrazolium bromide) assay. Human breast cancer cell lines (MCF-7) were pre-owned in this study. Cultured cells at concentration of 2000 cells/well was prepared and plated (100 $\mu \mathrm{L} /$ well) onto 96-well plates. The diluted sample extracts were added to each well with known concentrations; 50 , $150,250,350$ and $450 \mu \mathrm{g} / \mathrm{mL}$. The cells were then incubated for $24 \mathrm{~h}$ at 37 ${ }^{\circ} \mathrm{C}$ in a $5 \% \mathrm{CO}_{2}$ incubator. MTT solution was then added to each well and incubated for additional $3 \mathrm{~h}$ at $37{ }^{\circ} \mathrm{C}$ in the $5 \% \mathrm{CO}_{2}$ incubator. After solubilization of the purple formazan crystals using dimethyl sulfoxide (DMSO), the optical density (OD) of the well was measured using an ELISA reader at a wavelength of $570 \mathrm{~nm}$. The cytotoxicity was recorded as the drug concentration causing $50 \%$ growth inhibition of the tumor cells (IC 50 value) using the linear regression equation and an inhibition graph was plotted. The images of MCF-7 before and after treatment were also assessed by inverted microscope attached to a camera system.

$$
\% \text { Cell viability }=\frac{\text { OD of the Test sample (mean) }}{\text { OD of the Control (mean) }} \times 100
$$

\section{Results and Discussion}

Here we present the structural and morphological properties of PVP coated iron oxide nanoparticles and S. aromaticum loaded PVP coated iron oxide nanoparticles. The particles were characterized by UV-Vis spectroscopy, FTIR, DLS, and particle size analyzer, zeta potential, XRD and FT-Raman spectroscopy and its invitro cytotoxicity was studied by MTT assay method.

\subsection{Preparation of Iron Oxide Nanoparticles}

In this study, the $S$. aromaticum loaded PVP coated iron oxide nanoparticles were synthesized by wet chemical co-precipitation method which is adapted to make homogeneous nanoparticles more suitable for further study. In this protocol the matter of first importance is the visual characterization of the colour change pattern upon synthesis of nanoparticles. Here observed that the iron oxide nanoparticles manifest a colour change from light brown to thick brown. The colour change of the reaction mixture is due to addition of sodium hydroxide. 

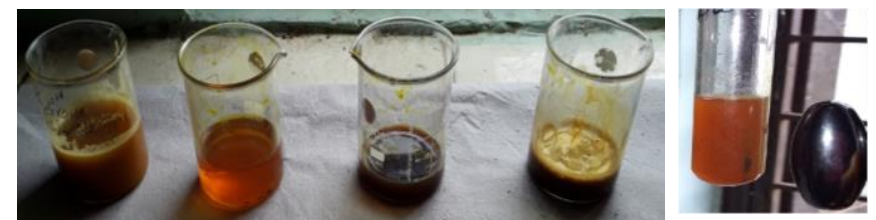

Fig. 1 The figure represents the colour change pattern upon synthesis of S.aromaticum loaded to PVP coated iron oxide NPs by wet chemical co-precipitation method and separation of synthesized NPs from the mixture using an external magnet

\subsection{UV-Visible Spectroscopy}

Formation of iron oxide nanoparticles were detected by spectral analysis under UV-Vis spectrophotometer. The range of the measurement is taken from 200 to $700 \mathrm{~nm}$. Deionised water was used as blank. Fig. 2a denotes the UV-Visible absorption spectra of PVP coated iron oxide and it showed an absorption peak at wave length $480 \mathrm{~nm}$. Fig. $2 \mathrm{~b}$ illustrates the absorbance of $S$. aromaticum loaded PVP coated iron oxide nanoparticles revealed a strong absorption at $554 \mathrm{~nm}$ due to surface plasmon resonance (SPR) to give respective peak. From the results, the UV-Vis spectrum analysis confirmed that the drug loading efficiency to the PVP coated iron oxide nanoparticles with the methanolic extract of the Syzygium aromaticum showed more efficiency. Similar types of results were observed using different plant extracts respectively [24, 25]. So, these results were correlated with previous observations.

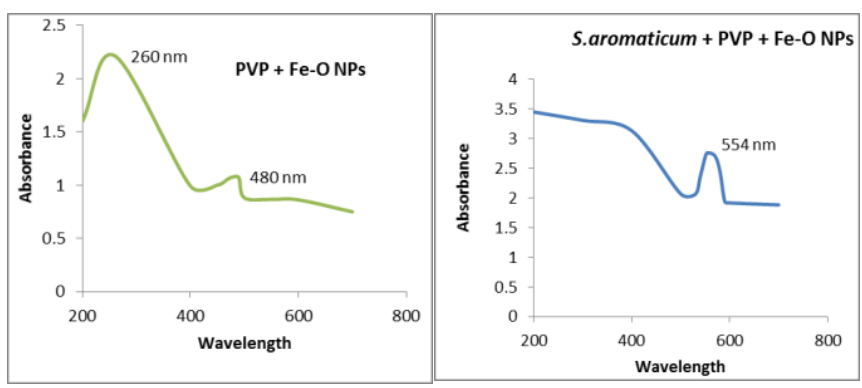

Fig. 2 UV-Visible spectroscopic studies of (a) PVP coated iron oxide nanoparticles and (b) S. aromaticum loaded iron oxide nanoparticles

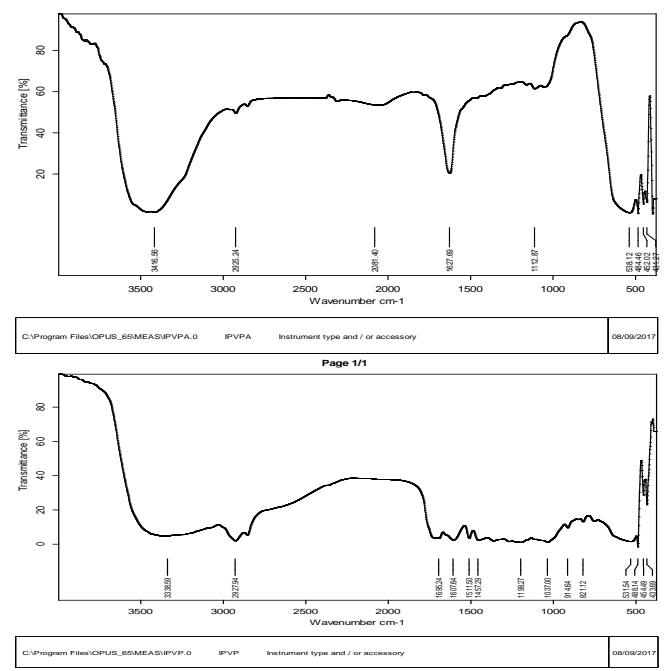

Fig. 3 FT-IR analysis of synthesized iron oxide nanoparticles (a) FTIR spectra of PVP coated iron oxide NPs and (b) FTIR spectra of S. aromaticum loaded to iron oxide NPs

\subsection{FTIR Analysis}

The crucial goal of FTIR spectroscopic analysis is to determine and identify the chemical functional groups present in the sample. IR spectroscopy is an important tool for identification, elucidation of iron oxide nanoparticles and to know the feasible phytochemicals responsible for capping and stabilization. FTIR spectra within $500-3500 \mathrm{~cm}^{-1}$ region were taken by mixing the samples with $\mathrm{KBr}$ powder. Fig. 3a represents the vibrational spectra of PVP coated iron oxide nanoparticles and exhibit strong and broad absorption peak at $3416 \mathrm{~cm}^{-1}$ corresponds to $0-\mathrm{H}$ stretching of alcohol and phenolic compounds and also confirmed the attachment of primary amines. It also showed carbonyl group characteristic absorption at $1627 \mathrm{~cm}^{-1}$. Fig $3 \mathrm{~b}$ shows the FTIR spectrum of S. aromaticum loaded PVP coated iron oxide nanoparticles. The strong absorption peak at $3338 \mathrm{~cm}^{-1}$ is assigned to $\mathrm{O}-\mathrm{H}$ bond and confirmed the https://doi.org/10.30799/jnst.178.19050103 attachment of primary amino group of drug on iron oxide nanoparticles. The presence of peak at $2927 \mathrm{~cm}^{-1}$ indicates aliphatic C-H stretching in methyl and methylene groups respectively. The peak at $1695 \mathrm{~cm}^{-1}$ is due to stretching vibration of $\mathrm{CO}$ groups in the ketones, aldehydes and carboxylic acids and it is attributed to the presence of carboxylate ions (COO-), which is responsible for the formation of iron oxide nanoparticles. The peak at $1037 \mathrm{~cm}^{-1}$ due to the presence of $\mathrm{CO}$ groups, which indicates the formation of iron oxide nanoparticles. The absorbance band at $821 \mathrm{~cm}$ ${ }^{1}$ might be assigned to the existence of some amount of oxidised iron oxide on the surface. Thus consequences confirmed that phenols and primary amines of plant extract were mainly responsible for capping and stabilization of iron oxide nanoparticles. This kind of results was obtained from iron oxide nanoparticles synthesized with the help of plantain peel extract [26].

\subsection{DLS Analysis}

The average particle size of $S$. aromaticum loaded PVP coated iron oxide nanoparticles were measured using a dynamic light-scattering particle size analyser. The average particle size of nanoparticles was shown in Fig. 4. The average particle size of PVP coated iron oxide nanoparticles was $399.8 \mathrm{~nm}$ and the mean particle size of $S$. aromaticum loaded PVP coated iron oxide nanoparticles was $190.1 \mathrm{~nm}$ respectively. DLS provided information about the hydrodynamic size and diameter of synthesized sample and it was found to be $1864 \mathrm{~nm}$ with a PDI of 0.946 for PVP coated iron oxide nanoparticles and $3445 \mathrm{~nm}$ with a PDI of 1.000 for $S$. aromaticum loaded to PVP coated iron oxide nanoparticles. In addition the results of particle size analysis from dynamic light scattering was synchronized with [27-30].

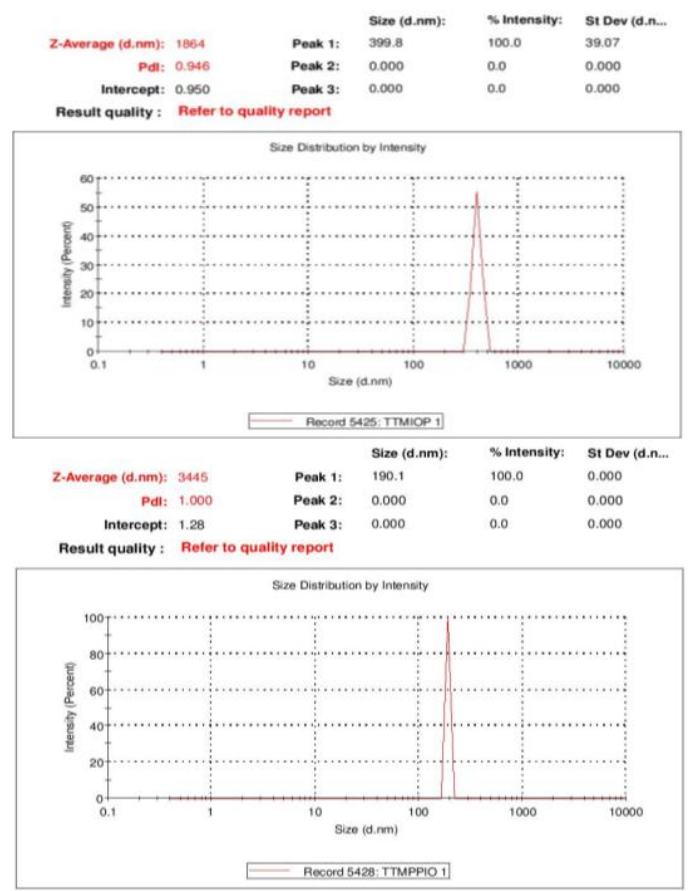

Fig. 4 DLS analysis of the samples (a) pvp coated iron oxide NPs and (b) $S$ aromaticum loaded iron oxide NPs

\subsection{Particle Size Analysis and Zeta Potential}

Recent investigations impressively show that the magnetic property of iron oxide based nanoparticles largely depends on particle size. The data was analysed based on intensity distribution. Fig. 5a indicates the polymer coated (PVP) iron oxide nanoparticles particle size as $254.1 \mathrm{~nm}$ and Fig. 5b represents $S$. aromaticum loaded PVP coated iron oxide nanoparticles mean size as $155.1 \mathrm{~nm}$. The drug loaded PVP coated iron oxide nanoparticles decreases in size when compared with PVP coated iron oxide nanoparticles this will be due to the coating of polymer to the iron oxide nanoparticles.

The stability of nanoparticles was monitored by long-term measurement of zeta potentials and relaxivity. Particles with zeta potentials more negative than $-30 \mathrm{mV}$ or more positive than $\pm 30 \mathrm{mV}$ were considered stable. The approximation to more negative zetapotentials ($30 \mathrm{mV}$ ) shows a tendency to an increase in particle stability [31]. The zeta potential values for the prepared PVP coated iron oxide nanoparticles and S. aromaticum loaded PVP coated iron oxide nanoparticles was measured and the values are observed to be $-23.7 \mathrm{mV}-18.8 \mathrm{mV}$ respectively as shown in Figs. 5 (c) and (d) and indicating a relative good stability of prepared nanoparticles. 


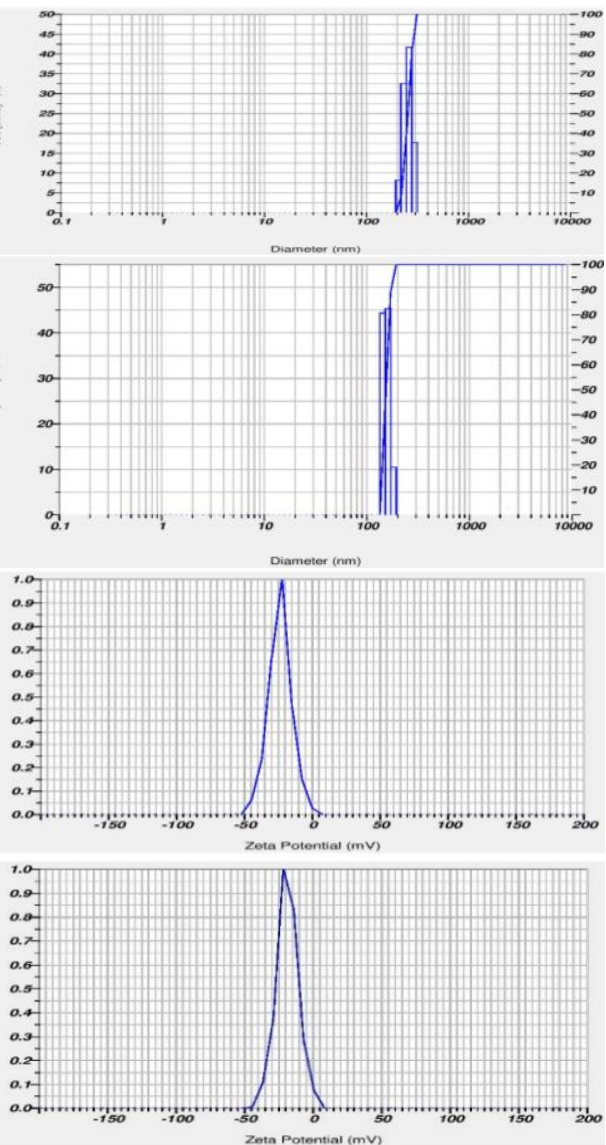

Fig. 5 Determination of the particle size and zeta potential analysis for the synthesized iron oxide NPs (a) PVP coated iron oxide NPs (b) S. aromaticum loaded iron oxide NPs (c) Zetapotential for PVP coated iron oxide NPs and (d) Zeta potential for $S$. aromaticum loaded iron oxide NPs

\subsection{XRD Studies}

The X-ray diffraction pattern of the iron oxide nanoparticles prepared with PVP coated iron oxide and S.aromaticum loaded iron oxide NPs are illustrated in Fig. 6(a,b). The characteristic peaks observed in PVP coated iron oxide NPs and $S$. aromaticum loaded iron oxide nanoparticles at different diffraction Bragg's angle $2 \theta=(30.22)$, (35.60), (45.60), (54.10), (57.68), (62.9) and (63.10) corresponding to the planes of diffraction peaks values (220), (311), (222), (400), (422), (440) and (511) respectively were analyzed by XRD pattern spectrum analysis using standards values of JCPDS card no. (19-0629).

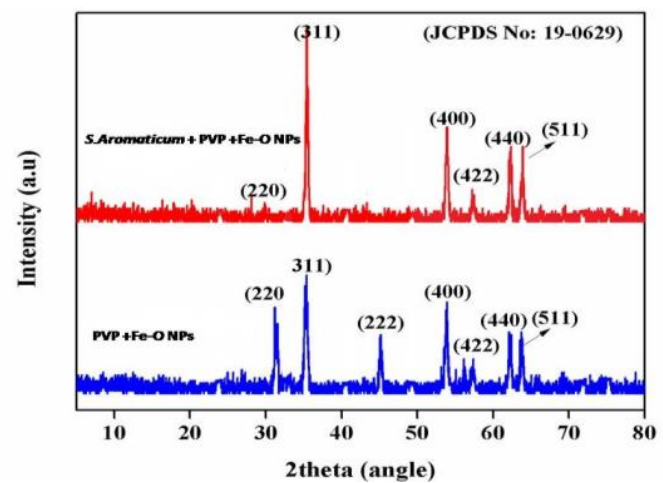

Fig. 6 XRD analysis of synthesized iron oxide NPs of (a)PVP coated iron oxide NPs and (b)drug loaded to PVP coated iron oxide NPs represents six Bragg reflections corresponding to end-centered monoclinic crystalline nature of nanoparticles

The above mentioned diffraction peaks illustrate the crystalline nature of the particles and the average crystalline diameter is to be estimated from X-ray line profile fitting, later it were found to be of the resulting nanoparticles was $32 \mathrm{~nm}$, which was calculated by the Debye-Scherrer equation and the average size of the individual magnetic nanoparticles is found to be about $8 \mathrm{~nm}$, the diffraction angle of the peak $\theta B=311$, and $D$ the average crystallite size for PVP coated iron oxide NPs and $S$. aromaticum loaded iron oxide NPs is $\beta$ (rad) $=23 \mathrm{~nm}$ and $29 \mathrm{~nm}$. XRD pattern of the samples represent diffused peaks at very high intensity at $35.60^{\circ}$ and corresponding to the iron oxide nanoparticles. The XRD peaks https://doi.org/10.30799/jnst.178.19050103 of the samples was consistent with standard XRD pattern of Fe-O (No: 190629) which confirmed the crystalline of iron oxide nanoparticles. The finding result was also confirmed with previous report [32-35].

\subsection{FT-Raman}

In the present work, Fig. 7 shows the spectrum of main features with well-defined dominant structural bands of the wave-numbers with vibration modes is observed near 229, 294, $410 \mathrm{~cm}^{-1}$ for the presence of PVP coated Fe-O NPs and 221,287, $404 \mathrm{~cm}^{-1}$ for the effect of $S$. aromaticum loaded iron oxide nanoparticles, whereas Raman peak shift is observed near 294, $410 \mathrm{~cm}^{-1}$ and 287 and $404 \mathrm{~cm}^{-1}$. So, for these samples, 3 main characteristic peaks are observed albeit with slight shift to the high wave number. In the Raman spectra of PVP coated iron oxide NPs and $S$. aromaticum loaded iron oxide nanoparticles observed small changes in the peak positions [36] have observed and assigned the bands from 200 to $600 \mathrm{~cm}^{-1}$ for iron oxide nanoparticles. The present investigation may also have related bands at the similar range are observed. Exposure of the samples to the laser power causes the bands to become better defined and shows better crystallization. This result suggests that the PVP coating may improve the stability of magnetic nanoparticles. In addition to, from the result all vibrational modes or peaks in PVP coated Fe-O NPs and $S$. aromaticum loaded Fe-O NPs are shifted due to change in crystallinity by adding the $S$. aromaticum. So, the drug is effectively changing the properties of iron oxide.

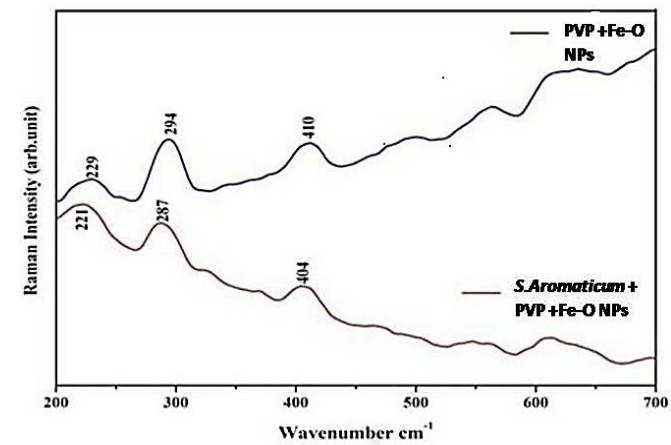

Fig. 7 Raman spectral data for synthesized iron oxide NPs (a) PVP coated iron oxide NPs and (b) S. aromaticum loaded to PVP coated iron oxide NPs

\subsection{Invitro Cytotoxicity Assay (MTT Assay)}

In this study, the primary measure illustrated to further strengthen the results and prove the capability and viability of PVP coated iron oxide nanoparticles and S. aromaticum loaded iron oxide nanoparticles as a potential anticancer agent for breast cancer by MTT assay on MCF-7 cell lines. As shown in Fig. 8, the cytotoxicity effect of the synthesized PVP coated iron oxide nanoparticles and S. aromaticum loaded iron oxide nanoparticles were investigated on MCF-7 cell lines. At $24 \mathrm{~h}$ of time course incubation period at $37^{\circ} \mathrm{C}$, a significant abatement in cell viability was observed in the treated cell lines, while the concentration of PVP coated iron oxide nanoparticles and $S$. aromaticum loaded to iron oxide nanoparticles was increased from 50,150,250, 350 and $450 \mu \mathrm{g} / \mathrm{mL}$. The standard graphs in MCF-7 cells for PVP coated iron oxide nanoparticles and S. aromaticum loaded iron oxide nanoparticles (Figs. 8 and 9) is plotted and from this graph, calculation of $\mathrm{IC}_{50}$ was done.

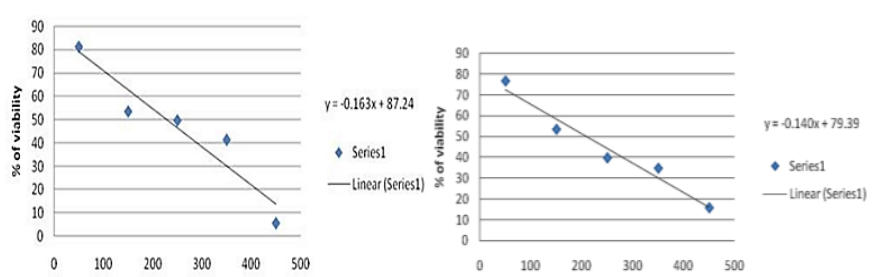

Fig. 8 Graph showing the percentage of inhibition at increased concentrations of PVP coated iron oxide NPs and S. aromaticum loaded to PVP coated iron oxide NPs

The microscopic observation of cells at increasing concentrations of PVP coated iron oxide nanoparticles and $S$. aromaticum iron oxide nanoparticles was done along with appropriate controls (Fig. 8). The MCF7 cell lines with their good morphology (C-) were lost with the addition of curcumin $(\mathrm{C}+)$. The morphology was altered with the addition of curcumin $(25 \mu \mathrm{g})$ which was used as a positive control. MCF-7 cell lines when treated with iron oxide nanoparticles inhibited proliferation of the cell lines in a dose-dependent and time-dependent manner. The morphology seems to be lost slightly with the treatment of PVP coated iron oxide nanoparticles and $S$. aromaticum iron oxide nanoparticles with the addition of $50 \mu \mathrm{g}$ and 
was further lost in an increasing order and finally the debris was found in the cells which are treated with $450 \mu \mathrm{g}$ by complete destruction of the cells (Fig. 8), indicating the anti-cancerous activity of PVP coated iron oxide nanoparticles and S. aromaticum iron oxide nanoparticles in those cells. However, there is a significant statistical difference in every condition when compared to negative control (C-) (Fig. 8).The number of live cells that were quantified from microscopic images (Fig. 8).

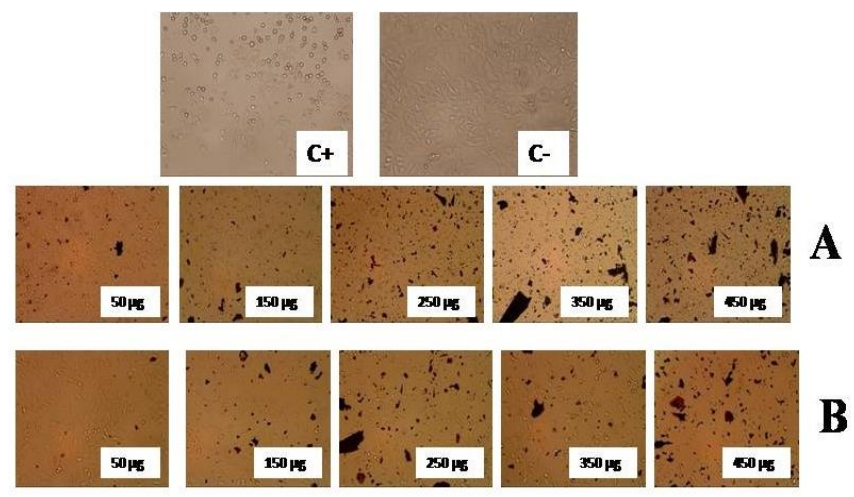

Fig. 9 Cell cytotoxicity assay in MCF-7 cells using PVP coated iron oxide nanoparticles and $S$. aromaticum loaded to PVP coated iron oxide nanoparticles

MTT cytotoxicity assay and $\mathrm{IC}_{50}$ determination were used for evaluating the extent of the anti-proliferative activity. The IC $\mathrm{C}_{50}$ value of PVP coated iron oxide nanoparticles and $S$. aromaticum loaded iron oxide nanoparticles may have the capability to reduce $50 \%$ of treated cell lines. The cytotoxicity of nanoparticles may depend on the small size and spherical shape of the particles [37]. The results showed when the concentration of the PVP coated iron oxide nanoparticles \& S. aromaticum loaded iron oxide nanoparticles and time of exposure was increased, the cell viability was decreased. These findings are significant because $S$. aromaticum loaded to iron oxide nanoparticles are selective towards cancer cells and are highly desirable feature of potential cancer chemo preventive and therapeutic agents. The previous report confirmed DOX loaded PVP coated iron oxide nanoparticles are promising magnetic drug carriers to be used in magnetically targeted drug delivery [38]. Besides these, there were many reports stating that the efficiency of the polymer coated iron oxide nanoparticles along with the drug molecule will act as the cell-specific delivery and kill the uptake cancer cells $[39,40]$.

\section{Conclusion}

Synthesis of iron oxide nanoparticles have been successfully synthesized by using an ecofriendly, economical, simple and fast method. A comprehensive study on their characterizations and pharmacognostic properties has been carried out. Our findings makes another proof that the stability of magnetic (Fe-O) nanoparticles increased with the addition of $S$. aromaticum extract. Further it also seems that $S$. aromaticum loaded PVP coated iron oxide nanoparticles are effective against breast cancer cell lines (MCF-7) as the MTT cytotoxicity assay has shown decrease in cell viability with the increase in drug concentration. Thus our present study proved that the $S$. aromaticum loaded iron oxide nanoparticles may lead to greater impact in the cancer clinical area as target based drug delivery besides recommending it for breast cancer in particular. Therefore it is concluded that $\mathrm{Fe}-\mathrm{O}$ NPs possess the versatility required to overcome some of the most challenging impediments in treatment of cancers.

\section{Acknowledgements}

The authors are thankful to DST-Curie Centre, Sri Padmavati Mahila Visvavidyalayam, Tirupati for extending the experimental facilities to carry out this work.

\section{References}

[1] R.L. Siegel, K.D. Miller, A. Jemal, Cancer statistics, Cancer J. Clinicians 66 (2016) 7-30.

[2] A.T. Toriola, G.A. Colditz, Trends in breast cancer incidence and mortality in the United States: implications of prevention, Breast Cancer Res Treat. 138(3) (2013) 665-673.

[3] J. Ferlay, I. Soerjomataram, M. Ervik, R. Dikshit, S. Eser, C. Mathers, Cancer incidence and mortality worldwide: IARC Cancer Base No.11. Lyon, France, 2013.
[4] G. Zhao, B.L. Rodriguez, Molecular targeting of liposomal nanoparticles to tumor microenvironment, Int. J. Nanomed. 8(1) (2013) 61-67.

[5] K.B. Sutradhar, M.L. Amin, Nanoemulsions: increasing possibilities in drug delivery, Europ. J. Nanomed. 5(2) (2013) 97-110.

[6] D. Peer, J.M. Karp, S. Hong, O.C. Farokhzad, R. Margalit, R. Langer, Nanocarriers as an emerging platform for cancer therapy, Nature Nanotech. 2(12) (2007) 751-760.

[7] N.J. Costa, L.M. Rossi, Synthesis of supported metal nanoparticle catalysts using ligand assisted methods, Nanoscale. 4 (2012) 5826-5834.

[8] P. Dauthal, M. Mukhopadhyay, Noble metal nanoparticles: plantmediated synthesis, mechanistic aspects of synthesis, and applications, Ind. Eng. Chem. Res. 55 (2016) 9557-9577.

[9] R.P. Chang, J. Yu, X. Ma, D.P. Anderson, Polysaccharides as stabilizers for the synthesis of magnetic nanoparticles, Carbohydr. Polym. 83(2) (2011) 640-644.

[10] K. Jia, R. Zhao, J. Zhong, X. Liu, Preparation and microwave absorption properties of loose nanoscale $\mathrm{Fe}_{3} \mathrm{O}_{4}$ spheres, J. Magn. Magn. Mater. 322 (2010) 2167-2171.

[11] D.K. Kim, Y. Zhang, W. Voit, K.V. Rao, J. Kehr, B. Bjelke, M. Mohamed, Superparamagnetic iron oxide nanoparticles for biomedical applications, Scr. Mater. 44 (2001) 1713-1717.

[12] A. Jordan, R. Scholz, P. Wust, H. Fahling, R.J. Felix, Magnetic fluid hyperthermia (MFH): cancer treatment with AC magnetic field induced excitation of biocompatible superparamagnetic nanoparticles, Magn. Magn. Mater. 201 (1999) 413-419.

[13] M.P. Vinardell, M. Mitjans, Antitumor activities of metal oxide nanoparticles, Nanomaterials. 5(2) (2015) 1004-1021.

[14] S.V.Jadhav, D.S. Nikam, V.M. Khot, N.D. Thorat, M.R. Phadatare, et al., Studies on colloidal stability of PVP-coated LSMO nanoparticles for magnetic fluid hyperthermia, New J. Chem. 37 (2013) 3121-3130.

[15] G. Lu, S. Li, Z. Guo, O.K. Farha, B.G. Hauser, et al., Imparting functionality to a metal- organic framework material by controlled nanoparticle encapsulation, Nat. Chem. 4 (2012) 310-316.

[16] H. Ziaei-Azad, N. Semagina, Bimetallic catalysts: Requirements for stabilizing PVP removal depend on the surface composition, Appl. Catal. A-Gen. 482 (2014) 327-335.

[17] A. Ruíz-Baltazar, R. Esparza, G. Rosas, R. Pérez, Effect of the surfactant on the growth and oxidation of iron nanoparticles, J. Nanomater. 240948 (2015) 1-8.

[18] C.M.K. Kumar, P. Yugandhar, N. Savithramma, Biological synthesis of silver nanoparticles from Adansonia digitata L. fruit pulp extract, characterization, and its antimicrobial properties. J. Intercult. Ethnopharmacol. 5 (2016) 79-85.

[19] B.D. Lade, A.S. Patil, Silver nano fabrication using leaf disc of Passiflora foetida Linn., Appl Nanosci. 7 (2017) 181-192.

[20] F. Liu, C. Liu, W. Liu, Z. Ding, H. Ma, et al., New sesquiterpenoids from Eugenia jambolana seeds and their anti-microbial activities, J. Agric Food Chem. 65 (2017) 10214-10222.

[21] S. Chanda, K. Nagani, In vitro and in vivo methods for anticancer activity evaluation and some Indian medicinal plants possessing anticancer properities: an overview, J. Pharm Phytochem. 2 (2013) 140-152.

[22] S. Hamedeyazdan, F. Fathiazad, S. Sharifi, H. Nazemiyeh, Antiproliferative activity of Marrubium persicum extract in the MCF-7 human breast cancer cell line, Asian. Pac. J.Cancer. Prev. 13 (2012) 5843-5848.

[23] V. Neveu, J. Perez-Jiménez, F. Vos, V. Crespy, L. du Chaffaut, L. Mennen, Phenolexplorer: an online comprehensive database on polyphenol contents in foods, Database 2010 (2010) bap024-1-9.

[24] P. Arsula Rose, P.K. Praseetha, M. Bhagat, P. Alexander, S. Abdeen, M. Chavali, Drug embedded PVP coated magnetic nanoparticles for targeted killing of breast cancer cells, Technol. Cancer Res. Treat. 12 (2013) 463-472.

[25] J.S. Swathy, P.K. Praseetha, G. Sakthivel, Targeted therapy for breast cancer cells by herbal drug formulations of iron oxide nanoparticles, Asian Jour. Pharm. Clinic. Res. 9 (2016) 347-353.

[26] S. Venkateswarlu, Y.S. Rao, T. Balaji, Biogenic synthesis of $\mathrm{Fe}_{3} \mathrm{O}_{4}$ magnetic nanoparticles using plantain peel extract, Mater. Lett. 100 (2013) 241-244.

[27] J.T. Cahil, J.N Ruppert, B. Wallis, Y. Liu, O.A. Graeve, Development of mesoporosity in scandia-stabilized zirconia: particle size, solvent and calcinations effects, Langmuir 30 (2014) 5585-5591.

[28] C.I. Vargas-consuelos, K. Seo, M. Camacho-Lopez, O.A. Graeve, Correlation between particle size and Raman vibrations in wo $_{3}$ powders, J. Phys. Chem. C 118 (2014) 9531-9537.

[29] O.A. Graeve, H. Fathi, J.P. Kelly, M.S. Saterlie, K. Sinha, et al., Reverse micelle synthesis of oxide nanopowders: mechanisms of precipitate formation and agglomeration effects, J. Colloid Interf. Sci. 407 (2013) 302-309.

[30] M.S. Saterlie, H. Sahin, B. Kavlicoglu, Y. Liu, O.A. Graeve, Surfactant effects on dispersion characteristics of copper-based nonofluids: a dynamic light scattering study, Chem. Mater. 24 (2012) 3299-3306.

[31] D.N. Williams, Surface-modified magnetic nanoparticles for cellular interactions and improved biological applications, Department of Chemical Engineering, University of Maryland, 2004.

[32] T.S. Mohammad, N.E. Mojtaba, S.E. Ali, E. Ehsan, Characterization of polymer coated iron nanoparticles, J. Chem. Chem. Eng. 14 (2011) 237-239.

[33] N. Basavegowda, K.B.S. Magar, K. Mishra, Green fabrication of ferromagnetic $\mathrm{Fe}_{3} \mathrm{O}_{4}$ nanoparticles and their novel catalytic applications for the synthesis of biologically interesting benzoxazinone and benzthioxazinone derivatives, New J. Chem. 38 (2014) 5415-5420.

[34] X.Y. Sun, S.S. Yu, J.Q. Wan, Facile graft of poly (2-methacryloyloxyethyl phosphorylcholine) onto $\mathrm{Fe}_{3} \mathrm{O}_{4}$ nanoparticles by ATRP: synthesis, properties and biocompatibility, J. Biomed. Mater. Res. A 101 (2013) 607-612.

[35] M. Ghassan Sulaiman, T. Amer Tawfeeq, S. Amal Naji, Biosynthesis, characterization of magnetic iron oxide nanoparticles and evaluations of the cytotoxicity and DNA damage of human breast carcinoma cell lines, Artificial cells, Nanomed. Biotechnol. (2017) 1366335 
[36] M. Mohammed Rahman, Sher Bahadar Khan, Aslam Jamal, Mohd Faisal, M. Abdullah Aisiri, Iron oxide nanoparticles, Nanomaterials, Prof. Mohammed Raman (Ed), Intechopen, Saudi Arabia, 2011.

[37] M.V. Park, A.M. Neigh, J.P. Vermeulen, L.J. De la Fonteyne, H.W. Verharen, J.J. Briede, The effect on particle size on the cytotoxicity, inflammation, development toxicity and genotoxicity of silver nanoparticles, Biomater. 32 (2011) 9810-9817.
[38] S. Kayal, R.V. Ramanujan, Doxorubicin loaded PVA coated iron oxide nanoparticles for targeted drug delivery, J. Mat. Sci. Eng. 30 (2010) 484-490.

[39] A. Sunitha, P.K. Praseetha, Diagnostics and treatment of metastatic cancers with magnetic nanoparticles, J. Nanomed. Biother. Discov. 3 (2013) 115-125.

[40] D. Jana, H. Radim, A. Vojtech, K. Rene, S. Oldrich, H. Jaromir, Preparation and properties of various magnetic nanoparticles, Sensors 6 (2009) 2352-2362. 